\title{
A classification for bicyclic and tricyclic graphs with respect to Tutte polynomial
}

\author{
Zahra Yarahmadi*(D), Shiva Mir(D) \\ Department of Mathematics, Faculty of Science, Khorramabad Branch, Islamic Azad University, \\ Khorramabad, I. R. Iran
}

\begin{abstract}
The Tutte polynomial of a graph is a polynomial in two variables defined for every simple graph contains information about how the graph is connected. We prove some formulas for computing Tutte polynomial of bicyclic and tricyclic graph and finally classify tricyclic graph with respect to Tutte polynomial.
\end{abstract}

Mathematics Subject Classification (2010). 05C05, 90B10

Keywords. Tutte polynomial, bicyclic, tricyclic graph

\section{Introduction}

Let $G$ be a simple graph. The vertex $v$ is reachable from another vertex $u$ if there is a path in $G$ connecting $u$ and $v$. In this case we write $v \alpha u$. A single vertex is a path of length zero and so $\alpha$ is reflexive. Moreover, we can easily prove that $\alpha$ is symmetric and transitive. So $\alpha$ is an equivalence relation on $V(G)$. The equivalence classes of $\alpha$ is called the connected components of $G$. A unicycle graph has only one cycle. An induced cycle means a cycle which is not contained another cycle as subgraph. A bicyclic graph and tricyclic graph mean, a graph contains two or three induced cycles, respectively. A bridge is an edge whose removal will cause the number of connected components to increase and a loop is an edge whose endpoints are the same vertex.

The Tutte polynomial of a graph $G, T(G ; x, y)$ defined by Tutte and Whitney is a polynomial in two variables defined for every simple graph contains information about how the graph is connected $[1,2,6,8-11]$. To define this concept, we need some notations. The edge contraction $G / u v$ of the graph $G$ is a graph is obtained by merging the vertices $u$ and $v$ and removing the edge $u v$. We write $G-u v$ for the graph where the edge $u v$ is merely removed, see Figure 1 . Then the Tutte polynomial of graph $G$ is defined by the recurrence relation between graph $G, G-u v$ and $G / u v$ as follows:

(i) If $e$ is neither a loop nor a bridge edge, then $T(G ; x, y)=T(G-e ; x, y)+$ $T(G / e ; x, y)$

(ii) If $e$ is a bridge edge, then $T(G ; x, y)=x T(G-e ; x, y)$,

(iii) If $e$ is a loop edge, then $T(G ; x, y)=y T(G-e ; x, y)$.

\footnotetext{
*Corresponding Author.

Email addresses: z.yarahmadi@gmail.com (Z. Yarahmadi), shivamir77@gmail.com (S. Mir)

Received: 10.03.2019; Accepted: 08.12.2019
} 


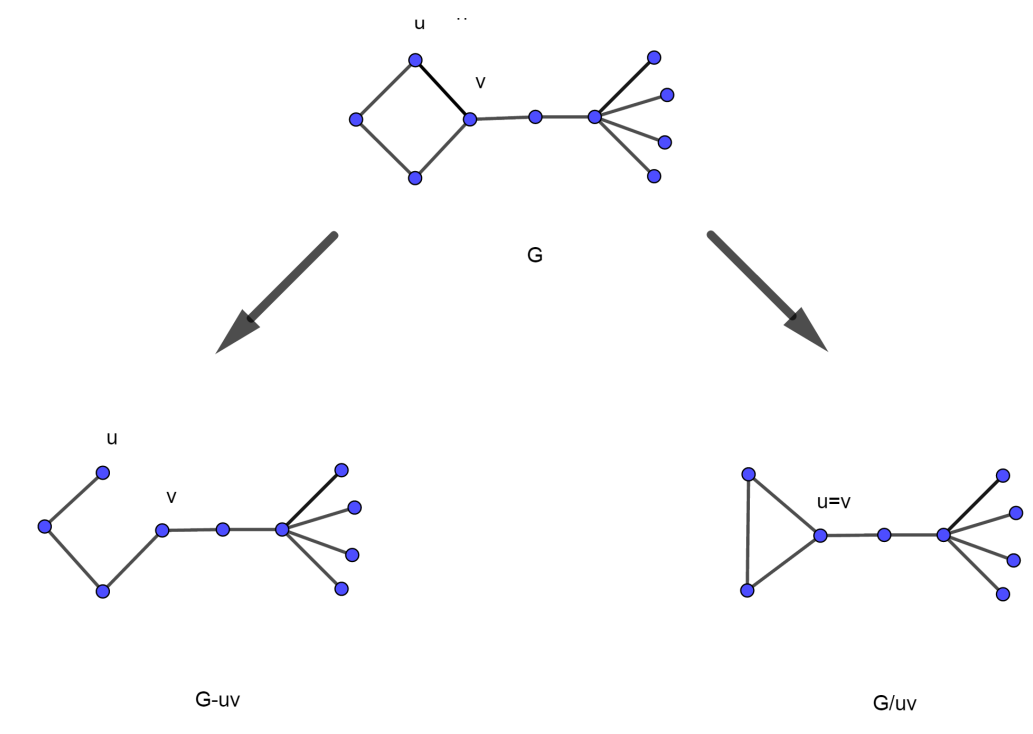

Figure 1. The Graph G, G-uv and G/uv.

If $G$ contained $i$ bridges and $j$ loops and $G^{\prime}$ is obtained by deleting of all bridges and loops of graph $G$, then $T(G ; x, y)=x^{i} y^{j} T\left(G^{\prime} ; x, y\right)$. If $G$ contains $i$ bridges and $j$ loops and no other edges, then $T(G ; x, y)=x^{i} y^{j}$. In particular, $T(G ; x, y)=1$ if $G$ contains no edges.

The importance of the Tutte polynomial $T(G ; x, y)$ comes from the algebraic graph theory as a generalization of counting problems dependent to graph coloring. It is also the source of several central computational problems in theoretical computer science. We note $T(G ; x, y)=T(G)$ briefly. In this paper we study on the Tutte polynomial of bicyclic and tricyclic graphs. In $[3-5,7]$. We classify bicyclic and tricyclic graph with respect to their Tutte polynomials. All over of this paper we assume that all graphs are simple.

\section{Main results}

In this section, at first we mention to the Tutte polynomial of special graph such as tree and cycle. Then by using these results continue our argument for all class of bicyclic and tricyclic graphs.

Lemma 2.1. Let $T_{n}$ be a tree with $n$ vertices. Then $T\left(T_{n}\right)=x^{n-1}$.

Proof. The proof is straightforward by definition of Tutte polynomial.

Lemma 2.2. Let $C_{n}$ be a cycle on $i$ vertices. Then $T\left(C_{n}\right)=\frac{x^{n}-x}{x-1}+y$.

Proof. Let $e$ be an edge of cycle $C_{n}$. By definition of Tutte polynomial, we have

$$
\begin{aligned}
T\left(C_{n}\right)= & T\left(C_{n}\right)-e+T\left(C_{n} / e\right) \\
= & T\left(P_{n}\right)+T\left(C_{n-1}\right) \\
= & x^{n-1}+T\left(P_{n-1}\right)+T\left(C_{n-2}\right) \\
& \vdots \\
= & x^{n-1}+x^{n-2}+\cdots+x^{4}+T\left(C_{3}\right) \\
= & x^{n-1}+x^{n-2}+\cdots+x^{4}+x^{3}+x^{2}+x+y \\
= & \frac{x^{n}-x}{x-1}+y .
\end{aligned}
$$


Remark 2.3. We note that in this paper $C_{1}$ means a loop, then $T\left(C_{1}\right)=y$. Also $C_{2}$ means a graph with two vertices and double edge between these vertices and it is easy to see that $C_{2}-e$ is a single edge and $C_{2} / e$ is a loop. Hence $T\left(C_{2}\right)=T\left(C_{2}-e\right)+T\left(C_{2} / e\right)=x+y$.

Theorem 2.4. Let $G$ be a connected graph with $t$ disjoint cycles of size $n_{1}, n_{2}, \cdots, n_{t}$. Then the Tutte polynomial of $G$ is

$$
T(G)=x^{b(G)} \prod_{i=1}^{t}\left(\frac{x^{n_{i}}-x}{x-1}+y\right)
$$

where $b(G)$ is the number of bridges of $G$.

Proof. We begin by deleting the bridges of the graph $G$. Suppose $B=\left\{e_{1}, e_{2}, \cdots, e_{b(G)}\right\}$ is the set of all bridges of $G$. Then we have:

$$
T(G)=x T\left(G-\left\{e_{1}\right\}\right)=x^{2} T\left(G-\left\{e_{1}, e_{2}\right\}\right)=\cdots=x^{b(G)} T\left(G-\left\{e_{1}, e_{2}, \cdots, e_{b(G)}\right\}\right) .
$$

Since all the cycles of the graph $G-\left\{e_{1}, e_{2}, \cdots, e_{b(G)}\right\}$ are disjoint, then by a well-known result we can conclude that $T\left(G-\left\{e_{1}, e_{2}, \cdots, e_{b(G)}\right\}\right)=\prod_{i=1}^{t}\left(\frac{x^{n_{i}}-x}{x-1}+y\right)$ and then $T(G)=x^{b(G)} \prod_{i=1}^{t}\left(\frac{x^{n_{i}}-x}{x-1}+y\right)$, which completes the proof.

Example 2.5. Let $G$ be a graph with three disjoint cycles, $C_{m}, C_{n}$ and $C_{l}$. All cases of position of cycles are shown in Figure 2. Then the Tutte polynomial of $G$ is obtained as, $T(G)=x^{b(G)} T\left(C_{m}\right) T\left(C_{n}\right) T\left(C_{l}\right)$.
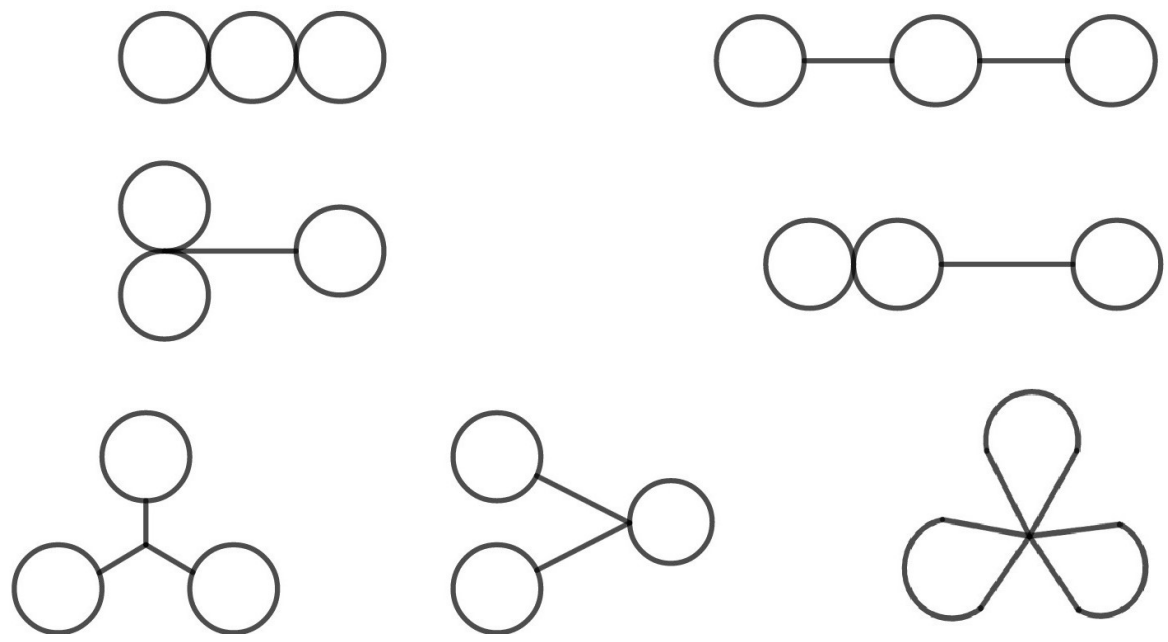

Figure 2. Different positions of cycles in the class of tricyclic graph with disjoint cycles.

Theorem 2.6. Let $G$ be a graph with two induced cycles, $C_{m}$ and $C_{n}$, with a common path $P_{t}$. Then, the Tutte polynomial of $G$ is obtained as follows:

$$
T(G)=x^{b(G)}\left(T\left(C_{m-t+1}\right) T\left(C_{n-t+1}\right)+\frac{x^{t-1}-x}{x-1} T\left(C_{m+n-2 t+2}\right)\right) .
$$

Proof. By Theorem 2.4., one can see that if $H$ is a graph, constructed by deleting bridges of $G$ then $T(G)=x^{b(G)} T(H)$. The graph $H$ is a graph constructed by two induced cycles, $C_{m}$ and $C_{n}$, such that they have a path $P_{t}$ in common, see Figure 3. Let $P_{t}: v_{1} e_{1} v_{2} e_{2} \cdots v_{t-1} e_{t-1} v_{t}$ be common path between $C_{m}$ and $C_{n}$. Then we have 


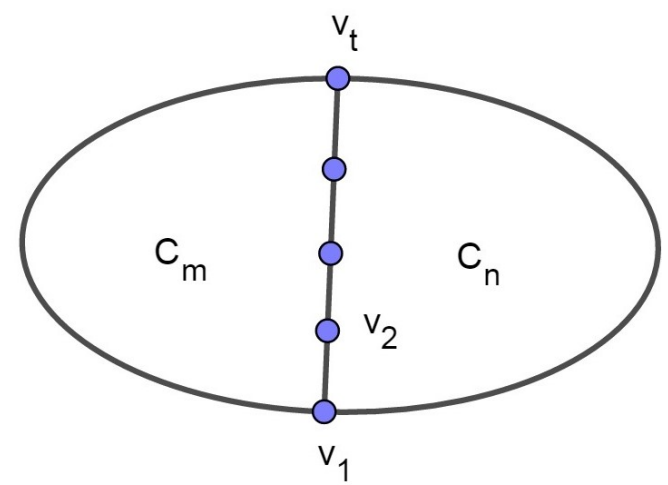

Figure 3. Graph $H$ with two induced cycles, $C_{m}$ and $C_{n}$.

$T(H)=T\left(H / e_{1}\right)+T\left(H-e_{1}\right)$. Since $H-e_{1}$ is a unicycle graph, then $T\left(H-e_{1}\right)=$ $x^{t-2} H\left(C_{m+n-2 t+2}\right)$. By continuing this process, we have

$$
\begin{aligned}
T(H)= & T\left(H / e_{1} / e_{2}\right)+T\left(H / e_{1}-e_{2}\right)+T\left(H-e_{1}\right) \\
= & T\left(H / e_{1} / e_{2} / e_{3}\right)+T\left(H / e_{1} / e_{2}-e_{3}\right)+T\left(H / e_{1}-e_{2}\right)+T\left(H-e_{1}\right) \\
& \vdots \\
= & T\left(H / e_{1} / e_{2} / \cdots / e_{t-1}\right)+T\left(H / e_{1} / e_{2} / \cdots / e_{t-2}-e_{t-1}\right) \\
& +T\left(H / e_{1} / e_{2} / \cdots / e_{t-3}-e_{t-2}\right)+\cdots+T\left(H / e_{1}-e_{2}\right)+T\left(H-e_{1}\right) \\
= & T\left(C_{m-t+1}\right) T\left(C_{n-t+1}\right)+T\left(C_{m+n-2 t+2}\right) \\
& +x T\left(C_{m+n-2 t+2}\right)+\cdots+x^{t-2} T\left(C_{m+n-2 t+2}\right) \\
= & T\left(C_{m-t+1}\right) T\left(C_{n-t+1}\right)+\frac{x^{t-1}-1}{x-1} T\left(C_{m+n-2 t+2}\right) .
\end{aligned}
$$

Since $T(G)=x^{b(G)} T(H)$, then

$$
T(G)=x^{b(G)}\left(T\left(C_{m-t+1}\right) T\left(C_{n-t+1}\right)+\frac{x^{t-1}-1}{x-1} T\left(C_{m+n-2 t+2}\right)\right),
$$

and the proof is completed.

Notation. Let $G$ be a graph which instructed with two induced cycles, $C_{m}$ and $C_{n}$, such that they have a path $P_{t}$ in common. We denote this graph by $G=G\left(C_{m}, C_{n}, P_{t}\right)$. By this notation, in Theorem 2.6, we have $T(G)=x^{b(G)} T\left(G\left(C_{m}, C_{n}, P_{t}\right)\right)$.

Corollary 2.7. Let $G$ be a tricyclic graph with two non-disjoint cycles $C_{m}, C_{n}$, with a path $P_{t}$ in common, and a disjoint cycle $C_{l}$, all cases of position of cycles are shown in Figure 4. The strict formula for Tutte polynomial of $G$ is $T(G)=x^{b(G)} T\left(G\left(C_{m}, C_{n}, P_{t}\right)\right) T\left(C_{l}\right)$.

Theorem 2.8. Let $G$ be a graph with three induced cycles, $C_{m}, C_{n}$ and $C_{l}$, such that $C_{m}$ and $C_{n}$ have a path $P_{s}$ in common, $C_{n}$ and $C_{l}$ have a path $P_{r}$ in common and $C_{l}$ and $C_{m}$ have a path $P_{t}$ in common. Then, the Tutte polynomial of $G$ is obtained as follows:

$$
\begin{aligned}
T(G)= & x^{b(G)}\left(T\left(C_{m-s-t+2}\right) T\left(G\left(C_{l-t+1}, C_{n-s+1}, P_{r}\right)\right)\right. \\
& +\frac{x^{t-1}-1}{x-1} T\left(G\left(C_{n-s+1}, C_{m+l-2 t-s+3}, P_{t}\right)\right) \\
& \left.+\frac{x^{s-1}-1}{x-1} T\left(G\left(C_{l}, C_{m+n-2 s+2}, P_{r+t-1}\right)\right)\right) .
\end{aligned}
$$



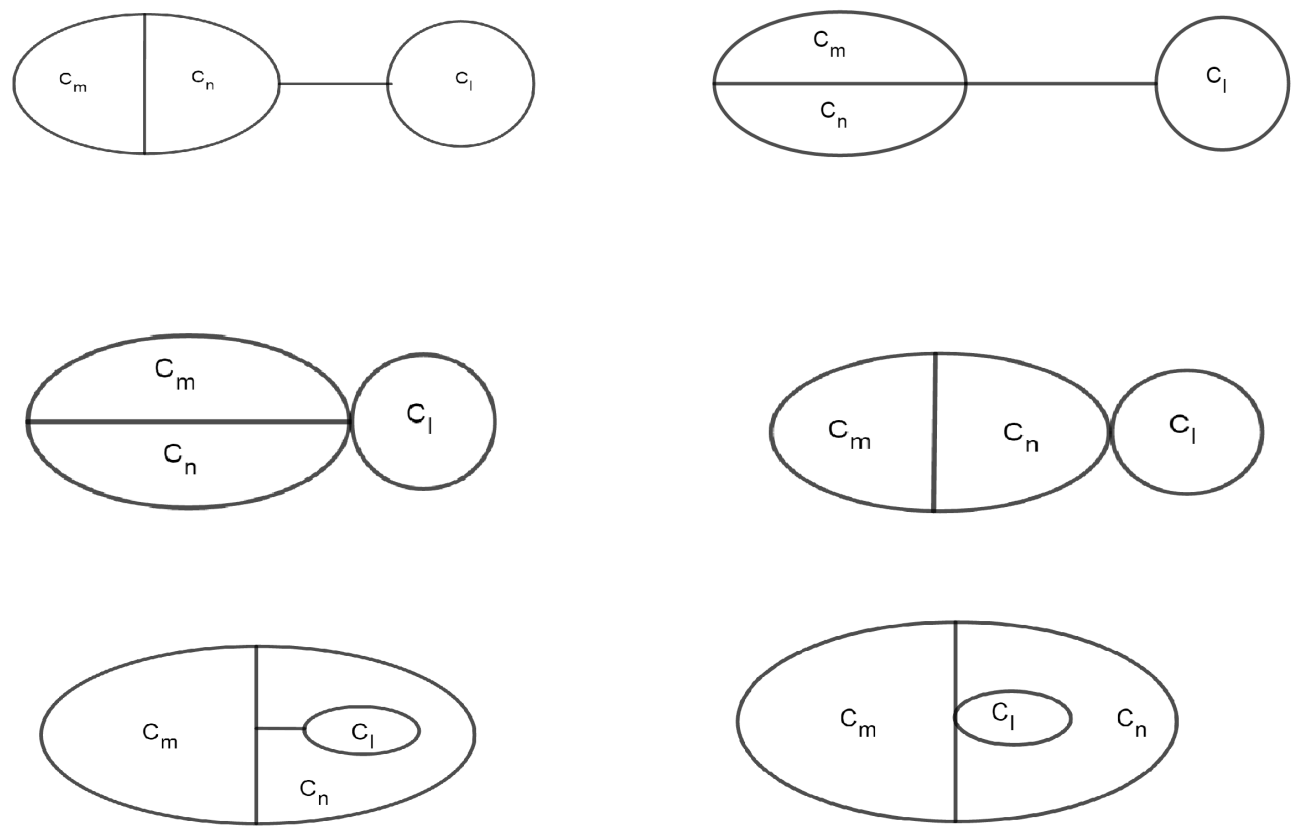

Figure 4. All positions of cycles of tricyclic graphs with two non-disjoint cycles and one disjoint cycle.

Proof. By well known result in Theorem 2.4., if $G$ has $b(G)$ bridge and $H$ obtained by deleting bridges of $G$, then $T(G)=x^{b(G)} T(H)$. See $H$ in Figure 5 .

Let $P_{s}: v_{1} e_{1} v_{2} e_{2} \cdots v_{s-1} e_{s-1} v_{s}$ be common path between $C_{m}$ and $C_{n}$. Then

$$
\begin{aligned}
T(H)= & T\left(H / e_{1} / e_{2}\right)+T\left(H / e_{1}-e_{2}\right)+T\left(H-e_{1}\right) \\
= & T\left(H / e_{1} / e_{2} / e_{3}\right)+T\left(H / e_{1} / e_{2}-e_{3}\right)+T\left(H / e_{1}-e_{2}\right)+T\left(H-e_{1}\right) \\
& \vdots \\
= & T\left(H / e_{1} / e_{2} / \cdots / e_{s-1}\right)+T\left(H / e_{1} / e_{2} / \cdots / e_{s-2}-e_{s-1}\right) \\
& +T\left(H / e_{1} / e_{2} / \cdots / e_{s-3}-e_{s-2}\right) \\
& \vdots \\
& +T\left(H / e_{1} / e_{2}-e_{3}\right)+T\left(H / e_{1}-e_{2}\right)+T\left(H-e_{1}\right) .
\end{aligned}
$$

For each $1 \leq i \leq s-2$, we have

$$
T\left(H / e_{1} / e_{2} / \cdots / e_{i}-e_{i+1}\right)=x^{s-i-2} T\left(G\left(C_{l}, C_{m+n-2 s+2}, P_{r+t-1}\right)\right) .
$$

Moreover $T\left(H-e_{1}\right)=x^{s-2} T\left(G\left(C_{l}, C_{m+n-2 s+2}, P_{r+t-1}\right)\right)$ and set $K=H / e_{1} / e_{2} / \cdots / e_{s-1}$. Hence

$$
T(H)=T(K)+\sum_{i=0}^{s-2} x^{s-i-2} T\left(G\left(C_{l}, C_{m+n-2 s+2}, P_{r+t-1}\right)\right) .
$$




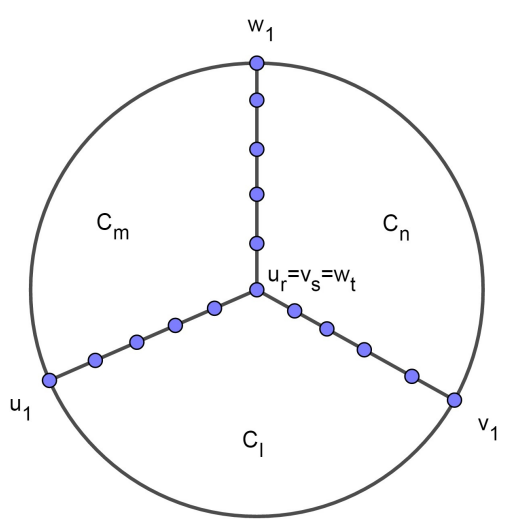

Figure 5. The Graph H.

On the other hands if $P_{t}: w_{1} e_{1}^{\prime} w_{2} e_{2}^{\prime} w_{t-1} e_{t-1}^{\prime} w_{t}$ be common path between $C_{m}$ and $C_{l}$, then

$$
\begin{aligned}
T(K)= & T\left(K / e_{1}^{\prime} / e_{2}^{\prime} / \cdots / e_{t-1}^{\prime}\right)+T\left(K / e_{1}^{\prime} / e_{2}^{\prime} / \cdots / e_{t-2}^{\prime}-e_{t-1}^{\prime}\right) \\
& +T\left(K / e_{1}^{\prime} / e_{2}^{\prime} / \cdots / e_{t-3}^{\prime}-e_{t-2}^{\prime}\right) \\
& \vdots \\
& +T\left(K / e_{1}^{\prime} / e_{2}^{\prime}-e_{3}^{\prime}\right)+T\left(K / e_{1}^{\prime}-e_{2}^{\prime}\right)+T\left(K-e_{1}^{\prime}\right) .
\end{aligned}
$$

We have $T\left(K / e_{1}^{\prime} / e_{2}^{\prime} / \cdots / e_{i}^{\prime}-e_{i+1}^{\prime}\right)=x^{t-i-2} T\left(G\left(C_{n-s+1}, C_{m+l-2 t-s+3}, P_{r}\right)\right)$ and $T\left(K-e_{1}^{\prime}\right)=x^{t-2} T\left(G\left(C_{n-s+1}, C_{m+l-2 t-s+3}, P_{r}\right)\right)$ for each $1 \leq i \leq t-2$.

Set $L=K / e_{1}^{\prime} / e_{2}^{\prime} / \cdots / e_{t-1}^{\prime}$, then $T(L)=T\left(C_{m-s-t+2}\right) T\left(G\left(C_{l-t+1}, C_{n-s+1}, P_{r}\right)\right)$. By the above argument, we conclude that

$$
\begin{aligned}
T(G)= & x^{b(G)}\left(T(K ; x, y)+\sum_{i=0}^{s-2} x^{s-i-2} T\left(G\left(C_{l}, C_{m+n-2 s+2}, P_{r+t-1}\right)\right)\right) \\
= & x^{b(G)}\left(T(L ; x, y)+\sum_{i=0}^{t-2} x^{t-i-2} T\left(G\left(C_{n-s+1}, C_{m+l-2 t-s+3}, P_{t}\right)\right)\right. \\
& \left.+\sum_{i=0}^{s-2} x^{s-i-2} T\left(G\left(C_{l}, C_{m+n-2 s+2}, P_{r+t-1}\right)\right)\right) \\
= & x^{b(G)}\left(T\left(C_{m-s-t+2}\right) T\left(G\left(C_{l-t+1}, C_{n-s+1}, P_{r}\right)\right)\right. \\
& +\frac{x^{t-1}-1}{x-1} T\left(G\left(C_{n-s+1}, C_{m+l-2 t-s+3}, P_{t}\right)\right) \\
& +\frac{x^{s-1}-1}{x-1} T\left(G\left(C_{l}, C_{m+n-2 s+2}, P_{r+t-1}\right)\right) .
\end{aligned}
$$

Theorem 2.9. Let $G$ be a graph with three induced cycles, $C_{m}, C_{n}$ and $C_{l}$, such that $C_{m}$ and $C_{n}$ have a path $P_{s}$ in common, $C_{n}$ and $C_{l}$ have a path $P_{r}$ in common, see Figure 6. Then, the Tutte polynomial of $G$ is obtained as follows:

$$
T(G)=x^{b(G)}\left(T\left(C_{s-1}\right) T\left(G\left(C_{n-s+1}, C_{l}, P_{r}\right)\right)+\frac{x^{m-s+1}-1}{x-1} T\left(G\left(C_{n}, C_{l}, P_{r}\right)\right)\right),
$$



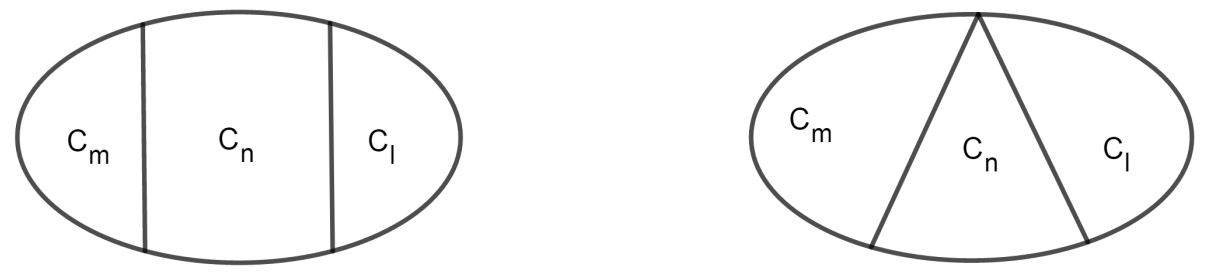

Figure 6. All positions of cycles of tricyclic graphs with conditions of Theorem 2.9.

where

$$
T\left(C_{s-1}\right)= \begin{cases}y & s=2 \\ x+y & s=3 .\end{cases}
$$

Proof. By similar reason of Theorem 2.8., one can see that $T(G)=x^{b(G)} T(H)$, without loosing generality assume that $H$ is a graph like Figure 7(a).

Suppose that $C_{m}: v_{1}, v_{2}, \cdots, v_{m-s+2}=u_{s}, u_{s-1}, \cdots, u_{1}=v_{1}$ and for $1 \leq i \leq m-s+1$, put $e_{i}=v_{i} v_{i+1}$.
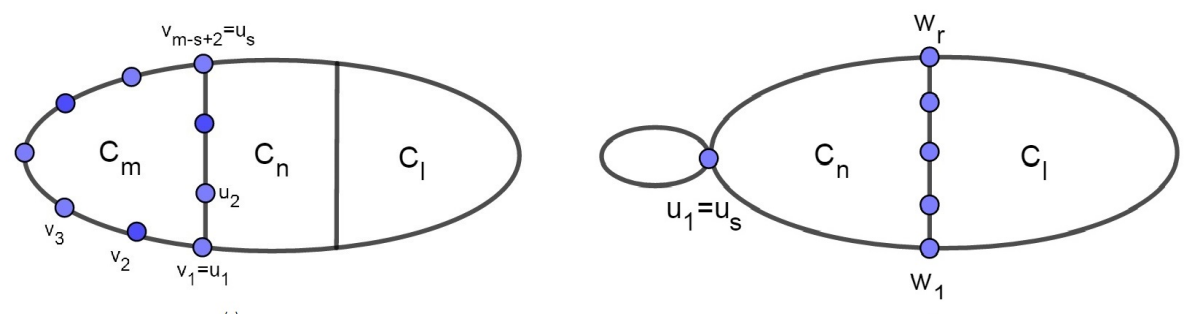

Figure 7. (a) The graph H, (b) The graph K.

Since $T(H)=T\left(H-e_{1}\right)+T\left(H / e_{1}\right)$, then by deleting each $e_{i}=v_{i} v_{i+1}$, remaining graph is a subgraph of $H$ contains two induced cycle $C_{n}$ and $C_{l}$ with a common path $P_{r}$. By using Theorem 4, we can obtain Tutte polynomial of $H$ as follows:

$$
\begin{aligned}
T(G)= & T\left(H-e_{1}\right)+T\left(H / e_{1}\right) \\
= & T\left(H / e_{1} / e_{2}\right)+T\left(H / e_{1}-e_{2}\right)+T\left(H-e_{1}\right) \\
= & T\left(H / e_{1} / e_{2} / e_{3}\right)+T\left(H / e_{1} / e_{2}-e_{3}\right)+T\left(H / e_{1}-e_{2}\right)+T\left(H-e_{1}\right) \\
& \vdots \\
= & T\left(H / e_{1} / e_{2} / \cdots / e_{m-s+1}\right)+T\left(H / e_{1} / e_{2} / \cdots / e_{m-s}-e_{m-s+1}\right) \\
& +T\left(H / e_{1} / e_{2} / \cdots / e_{m-s-1}-e_{m-s}\right) \\
& \vdots \\
& +T\left(H / e_{1} / e_{2}-e_{3}\right)+T\left(H / e_{1}-e_{2}\right)+T\left(H-e_{1}\right) .
\end{aligned}
$$

For each $1 \leq i \leq m-s, T\left(H / e_{1} / e_{2} / e_{3} / \cdots / e_{i}-e_{i+1}\right)=x^{m-s-i} T\left(G\left(C_{n}, C_{l}, P_{r}\right)\right)$ and $T\left(H / e_{1}\right)=x^{m-s} T\left(G\left(C_{n}, C_{l}, P_{r}\right)\right)$. Set $K=T\left(H / e_{1} / e_{2} / \cdots / e_{m-s+1}\right)$, see Figure $7(\mathrm{~b})$. 
On the other hand, $T(K)=T\left(C_{s-1}\right) T\left(G\left(C_{n-s+1}, C_{l}, P_{r}\right)\right)$. Hence, by above argument, one can see that:

$$
\begin{aligned}
T(H) & =T\left(C_{s-1}\right) T\left(G\left(C_{n-s+1}, C_{l}, P_{r}\right)\right)+\sum_{i=1}^{m-s} x^{m-s-i} T\left(G\left(C_{n}, C_{l}, P_{r}\right)\right)+x^{m-s} T\left(G\left(C_{n}, C_{l}, P_{r}\right)\right) \\
& =T\left(C_{s-1}\right) T\left(G\left(C_{n-s+1}, C_{l}, P_{r}\right)\right)+\frac{x^{m-s+1}-1}{x-1} T\left(G\left(C_{n}, C_{l}, P_{r}\right)\right)
\end{aligned}
$$

and this completes the proof.

Theorem 2.10. Let $G$ be a graph with three induced cycles, $C_{m}, C_{n}$ and $C_{l}$, such that $C_{l}$ and $C_{n}$ have a path $P_{t}$ in common and the path $P_{s}$ is common between three cycles $C_{m}$, $C_{n}$ and $C_{l}$, Figure 8. Then, the Tutte polynomial of $G$ is obtained as follows:

$$
T(G)=x^{b(G)}\left(T\left(C_{s-1}\right) T\left(G\left(C_{n-s+1}, C_{l-s+1}, P_{t-s+1}\right)\right)+\frac{x^{m-s+1}-1}{x-1} T\left(G\left(C_{n}, C_{l}, P_{t}\right)\right)\right)
$$
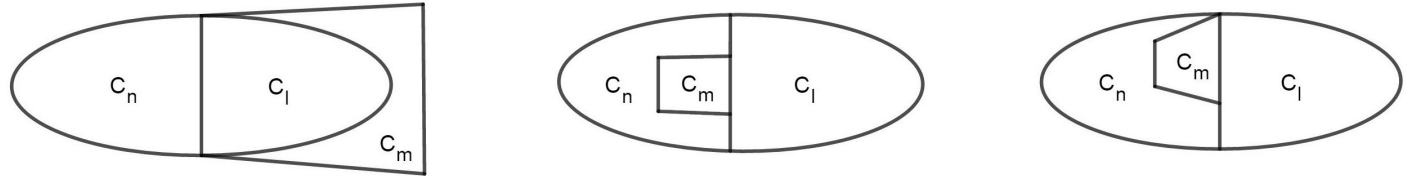

Figure 8. All positions of cycles of tricyclic graphs with conditions of Theorem 2.10.

Proof. By similar way of Theorem 2.8 and $2.9, T(G)=x^{b(G)} T(H)$. Without loosing of generality $H$ is shown in Figure 9(a).

Suppose that $C_{m}: v_{1}, v_{2}, \cdots, v_{m-s+2}=u_{s}, u_{s-1}, \cdots, u_{1}=v_{1}$ and for $1 \leq i \leq m-s+1$, put $e_{i}=v_{i} v_{i+1}$.
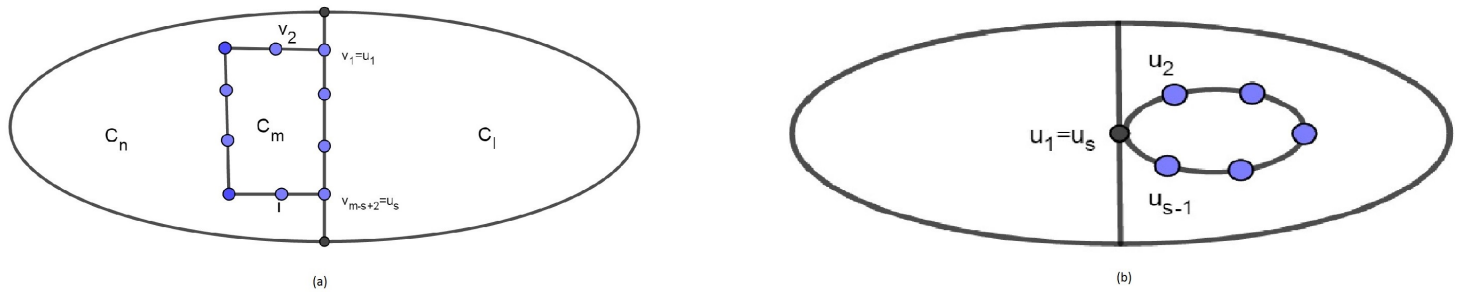

Figure 9. (a) The Graph H, (b) The Graph K.

Since $T(H)=T\left(H-e_{1}\right)+T\left(H / e_{1}\right)$, then by deleting each $e_{i}=v_{i} v_{i+1}$, remaining graph is a subgraph of $H$ contains two induced cycle $C_{n}$ and $C_{l}$ with a common path $P_{r}$. By using Theorem 2.6., we can obtain Tutte polynomial of $H$ as follows:

$$
T(H)=T\left(H / e_{1} / e_{2} / e_{3} / \cdots / e_{m-s+1}\right)+\sum_{i=1}^{m-s} T\left(H / e_{1} / e_{2} / e_{3} / \cdots / e_{i}-e_{i+1}\right)+T\left(H-e_{1}\right) .
$$

For each $1 \leq i \leq m-s, T\left(H / e_{1} / e_{2} / e_{3} / \cdots / e_{i}-e_{i+1}\right)=x^{m-s-i} T\left(G\left(C_{n}, C_{l}, P_{r}\right)\right)$ and $T\left(H / e_{1}\right)=x^{m-s} T\left(G\left(C_{n}, C_{l}, P_{r}\right)\right)$. Set $K=T\left(H / e_{1} / e_{2} / \cdots / e_{m-s+1}\right)$, see Figure 9 (b). It is easy to see that, $T(K)=T\left(C_{s-1}\right) T\left(G\left(C_{n-s+1}, C_{l-s+1}, P_{t-s+1}\right)\right)$. 
Hence, by above argument, one can see that:

$$
\begin{aligned}
T(H) & =T\left(C_{s-1}\right) T\left(G\left(C_{n-s+1}, C_{l-s+1}, P_{t-s+1}\right)\right)+\sum_{i=0}^{m-s} x^{m-s-i} T\left(G\left(C_{n}, C_{l}, P_{t}\right)\right) \\
& =T\left(C_{s-1}\right) T\left(G\left(C_{n-s+1}, C_{l-s+1}, P_{t-s+1}\right)\right)+\frac{x^{m-s+1}-1}{x-1} T\left(G\left(C_{n}, C_{l}, P_{t}\right)\right) .
\end{aligned}
$$

This completes the proof.

\section{Conclusion remarks}

In this paper some strict formulas of bicyclic and tricyclic graphs are obtained. We also characterize different classes of bicyclic and tricyclic graph by Tutte polynomial. We classify bicyclic graphs in to two different classes and tricyclic graphs in five different classes with respect to Tutte polynomial and collect all results in Figures 10-13.
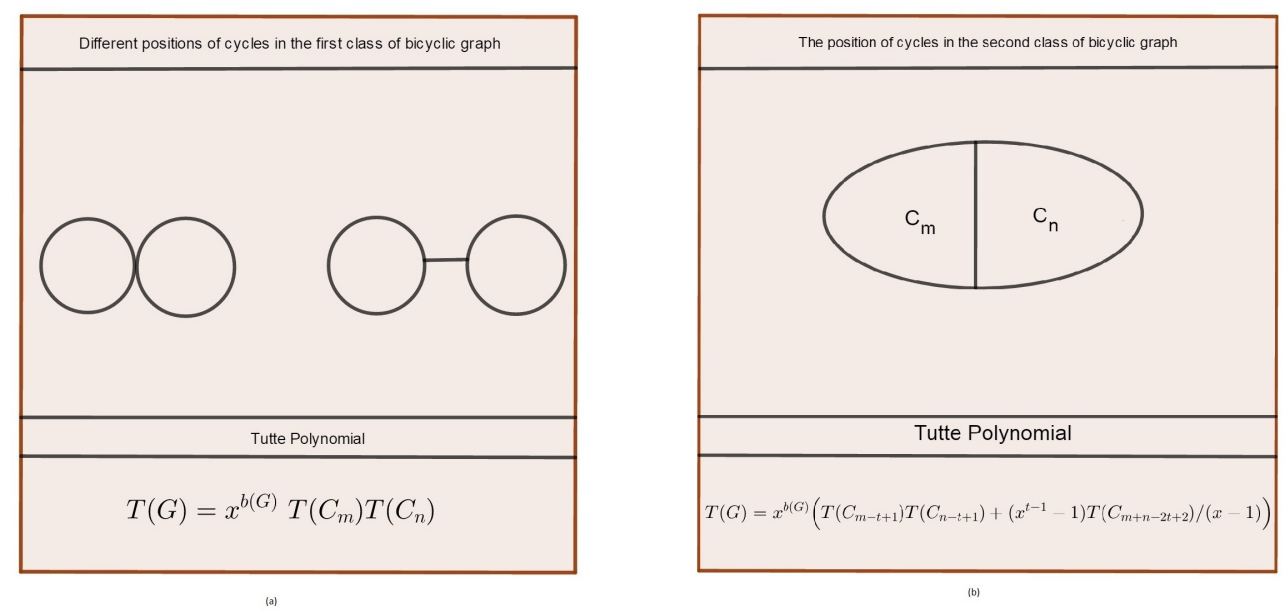

Figure 10. (a) First class of bicyclic graph with the same Tutte polynomial, (b) Second class of bicyclic graph with respect to Tutte polynomial.
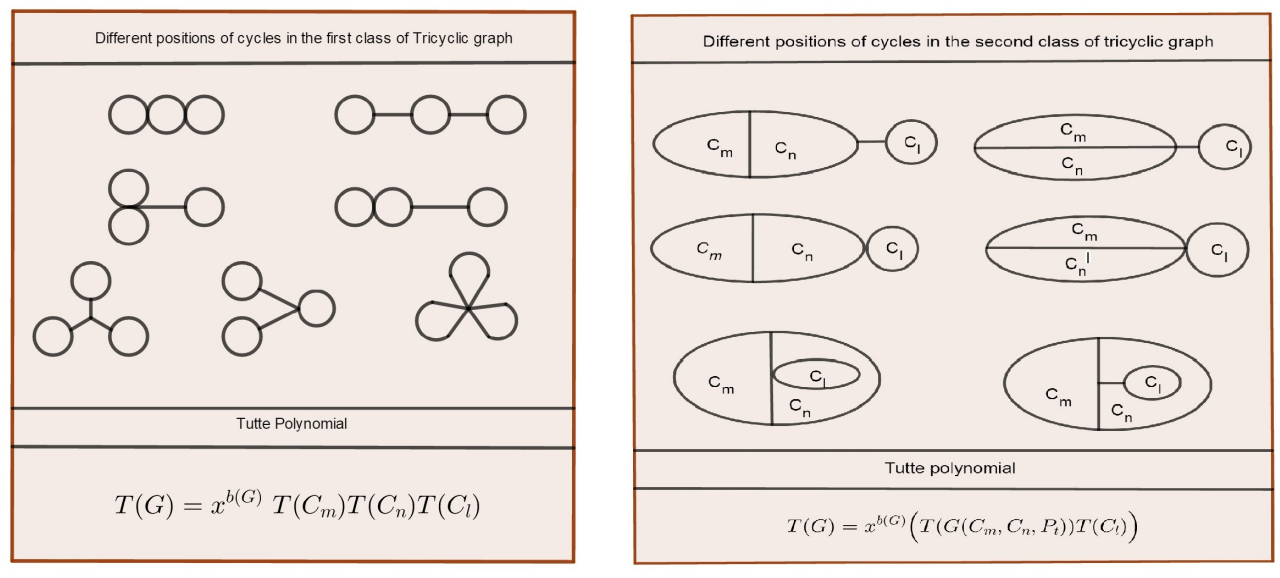

Figure 11. (a) First class of tricyclic graph with the same Tutte polynomial, (b) Second class of tricyclic graph with the same Tutte polynomial. 

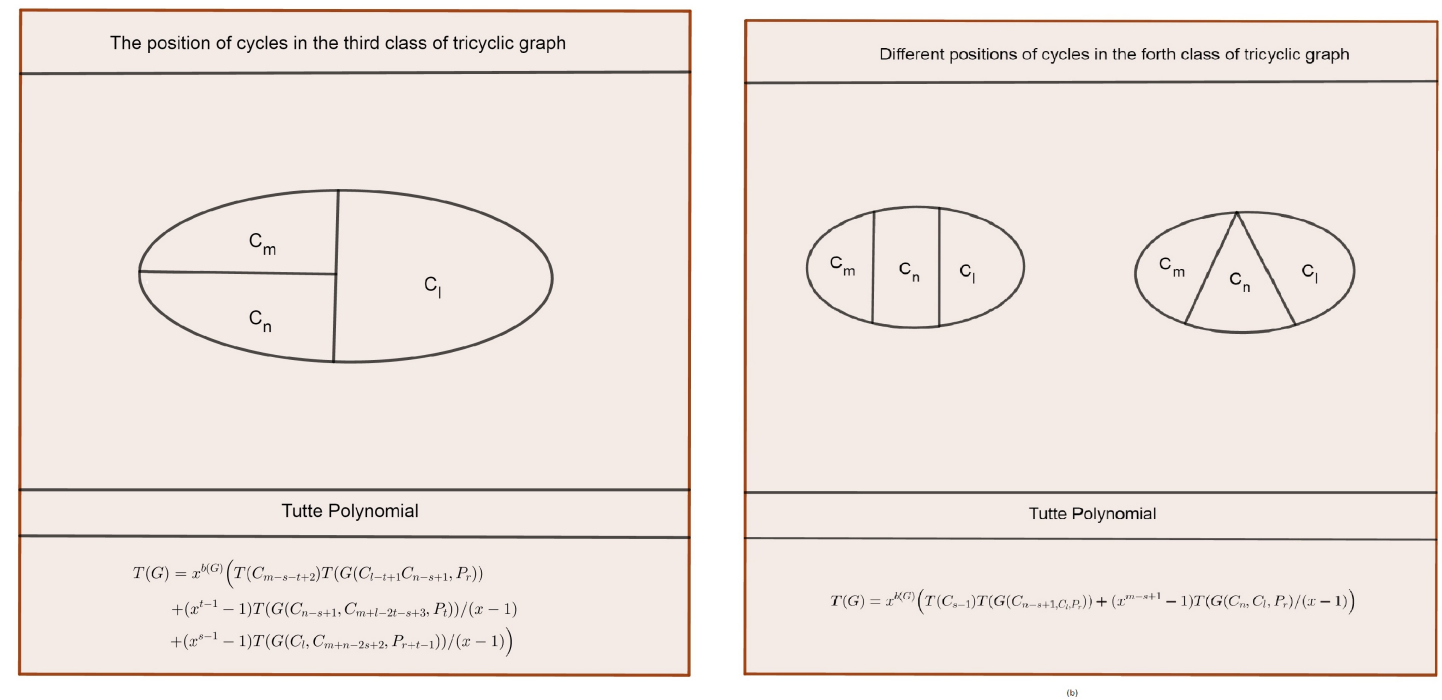

Figure 12. (a) Third class of tricyclic graph with respect to Tutte polynomial, (b) Forth class of tricyclic graph with respect to Tutte polynomial.

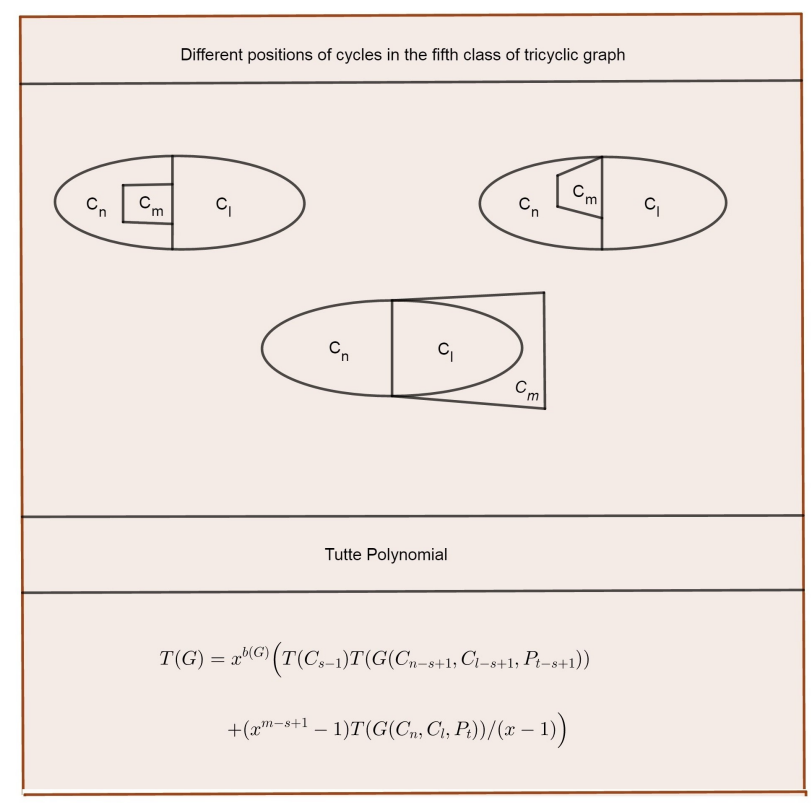

Figure 13. Fifth class of tricyclic graph with respect to Tutte polynomial.

\section{References}

[1] J.M. Burgos, Singularities in Negamis splitting formula for the Tutte polynomial, Discrete Appl. Math. 237, 65-74, 2018.

[2] H.H. Crapo, The Tutte polynomial, Aequationes Math. 3 (3), 211-229, 1969.

[3] H. Deng, S. Chen and J. Zhang, The Merrifield-Simmons index in(n, n+1)-graphs, J. Math. Chem. 43, 75-91, 2008.

[4] A. Dolati, M. Haghighat, S. Golalizadeh and M. Safari, The smallest Hosoya index of con-nected tricyclic graphs, MATCH Commun. Math. Comput. Chem. 65, 57-70, 2011. 
[5] S. Li and Z. Zhu, Sharp lower bound for total number of matching of tricyclic graphs, Electron. J. Comb. 17, 15 pages, 2010.

[6] S. Ok and T.J. Perrett, Density of real zeros of the Tutte polynomial, Electron. J. Discrete Math. 61, 941-946, 2017.

[7] Y.M. Tong, J.B. Liu, Z.Z. Jiang and N.N. Lv, Extreme values of the first general Zagreb index in tricyclic graphs, J. Hefei Univ. Nat. Sci. 1, 4-7, 2010.

[8] W.T. Tutte, A contribution to the theory chromatic polynomials, Canad. J .Math. 6, 80-91, 1953.

[9] W.T. Tutte, On dichromatic polynomials, J. Combin. Theory 2, 301-320, 1967.

[10] W.T. Tutte, On chromatic polynomials and the golden ratio, J. Combin. Theory 9, 289-296, 1970.

[11] W.T. Tutte, Graph-polynomials, Adv. Appl. Math. 32, 5-9, 2004. 